EPJ Web of Conferences 70, 00052 (2014)

DOI: $10.1051 /$ epjconf/ 20147000052

(C) Owned by the authors, published by EDP Sciences, 2014

\title{
The HERA Proton
}

\author{
Shiraz Habib \\ On behalf of the $\mathrm{H} 1$ and ZEUS Collaborations \\ e-mail: shiraz.habib@desy.de \\ ${ }^{1}$ Deutsches Elektronen-Synchrotron (DESY) \\ Notkestrasse 85 \\ 22607 Hamburg
}

\begin{abstract}
The almost $1 \mathrm{fb}^{-1}$ of $e p$ data collected by the H1 and ZEUS collider experiments at HERA allows for a precise determination of the proton's parton distribution functions (PDFs). Measurements used to constrain the PDFs - inclusive and jet cross sections, charm contribution to the $F_{2}$ proton structure function, $F_{2}^{c \bar{c}}-$ are presented herein. The measurement process itself includes cataloguing the sensitivity of the cross sections to the various sources of correlated systematic uncertainties. In the jet measurement, correlations of a statistical nature are also quantified and catalogued. These correlations provide a basis to combine measurements of the same physical observable across different time periods, experiments and measurement methodology. The subsequent PDF fitting procedure also takes into account such correlations. The resulting HERAPDF1.5 set based on inclusive data as well as PDF sets derived from inclusive plus charm data are presented togeteher with their predictions for $p p$ cross sections at the LHC.
\end{abstract}

\section{Introduction}

The complete $1 \mathrm{fb}^{-1}$ ep HERA data set included both electron and positron beams at $27.6 \mathrm{GeV}$ and proton beams at $920,820,575$ and $460 \mathrm{GeV}$. The lepton beam polarization ${ }^{1} P_{e}$, ranged between $-1 / 3$ $\lesssim P_{e} \lesssim+1 / 3$ including ensemble states of $P_{e}=0$. The volume of data together with the rich mix of initial states provide tight constraints on different PDF sensitivities.

The H1 and ZEUS experiments housed at HERA typically measured the same cross sections over time using different experimental methods. These measurements when combined by using a $\chi^{2}$ minimization method [1] which takes into account correlations between different sources of systematic uncertainty, results in significant improvements in both statistical as well as systematic errors. The combined cross sections form a well understood consistent data set. This allows its use as a constraint on the PDFs of a parametrized proton with the conventional $\chi^{2}$ tolerance of $\Delta \chi^{2}=1$.

\section{Results}

The unpolarized NC and CC HERA I measurements of H1 and ZEUS are combined[2]. The data span six orders of magnitude in negative four-momentum transfer squared $Q^{2}$ and Bjorken- $x$. Altogether

\footnotetext{
${ }^{1}$ Defined as the fraction of the beam that was right-handed minus the left-handed fraction.
} 
1 a

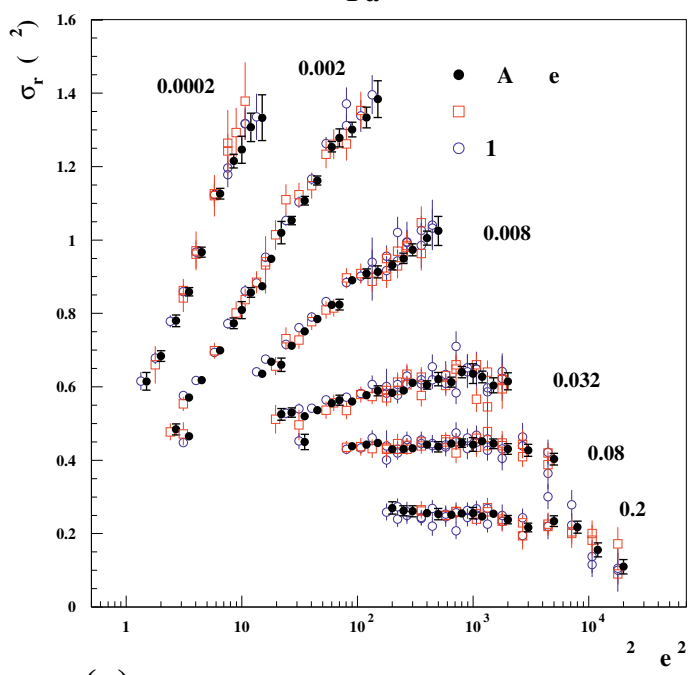

(a)

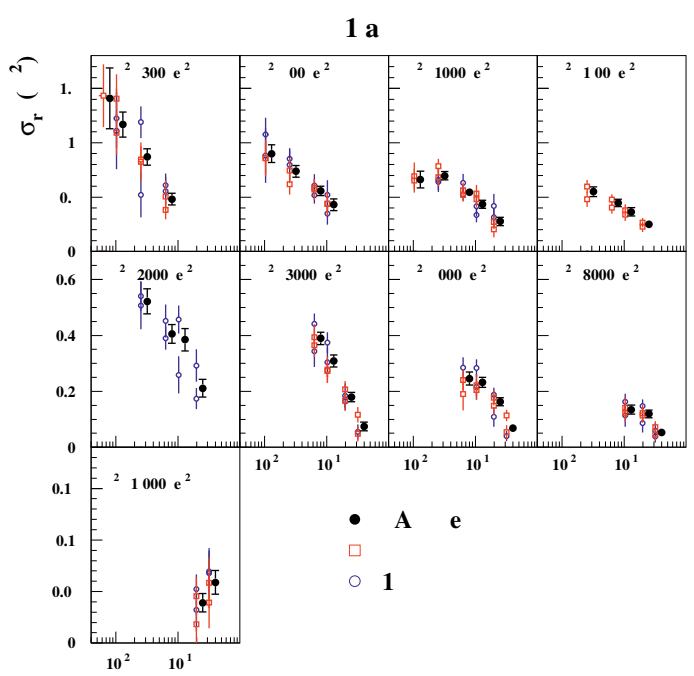

(b)

Figure 1. (a) HERA combined NC $e^{+} p$ reduced cross section as a function of $Q^{2}$ for six $x$-bins compared to the separate $\mathrm{H} 1$ and ZEUS data input to the combination procedure. The individual measurements are displaced horizontally for better visibility. (b) HERA combined $\mathrm{CC} e^{+} p$ reduced cross section compared to the separate $\mathrm{H} 1$ and ZEUS data input to the averaging procedure. The individual measurements are displaced horizontally for better visibility.

1402 measurements with 110 correlated sources of uncertainty are combined to 741 cross sections giving a $\chi^{2} /$ ndf of $636.5 / 656$. Figure 1 (a) shows the $\mathrm{NC} e^{+} p$ reduced cross section as a function of $Q^{2}$ for six $x$ bins. Shown are the individual H1 and ZEUS measurements as well as the combined measurement. As can be seen, the precision of the measurement improves significantly after the combination. The overall precision of the $\mathrm{NC}$ cross sections is $2 \%$ for $3<Q^{2}<500 \mathrm{GeV}^{2}$ and reaches $1 \%$ for $20<Q^{2}<100 \mathrm{GeV}^{2}$. Figure 1 (b) shows the $\mathrm{CC} e^{+} p$ reduced cross section as a function of $x$ for at fixed $Q^{2}$ values.

The combined inclusive data from HERA I is used as the sole data input to a QCD analysis to produce the HERAPDF1.0 set. The evolution of the PDFs is done at NLO at the starting scale of $Q^{2}=1.9 \mathrm{GeV}^{2}$ using the DGLAP evolution scheme [9-12]. The data is restricted to $Q^{2} \geqslant 3.5$ $\mathrm{GeV}^{2}$. Subsequently a QCD analysis is performed using the combined inclusive data from HERA I as well as from high $Q^{2}$ HERA II to produce the HERAPDF1.5 set. In the QCD analysis, the evolution of the PDFs is done at NLO [3] and NNLO [4] using a similar fitting procedure to that of HERAPDF1.0. The valence quarks, sea quarks and gluon distributions are shown for HERAPDF1.0 (at $Q^{2}=1.9 \mathrm{GeV}^{2}$ ) and the NLO version of HERAPDF1.5 (at $Q^{2}=2 \mathrm{GeV}^{2}$ ) in Figures 2(a) and (b) respectively. The precision of the PDFs at high-x is considerably improved in HERAPDF1.5 compared to HERAPDF1.0, particularly in the valence sector. The corresponding $\mathrm{W}$ and $\mathrm{Z}$ cross section predictions based on this PDF set have uncertainties of a few percent, comparable to the uncertainties from CTEQ and MSTW. 


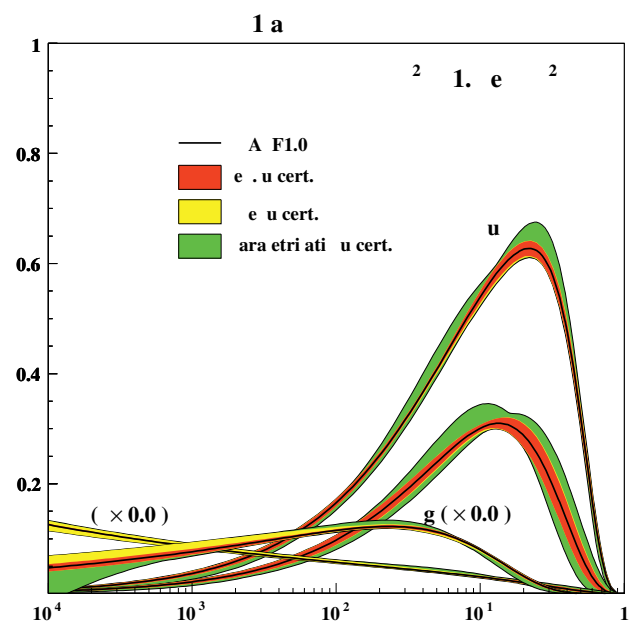

(a)

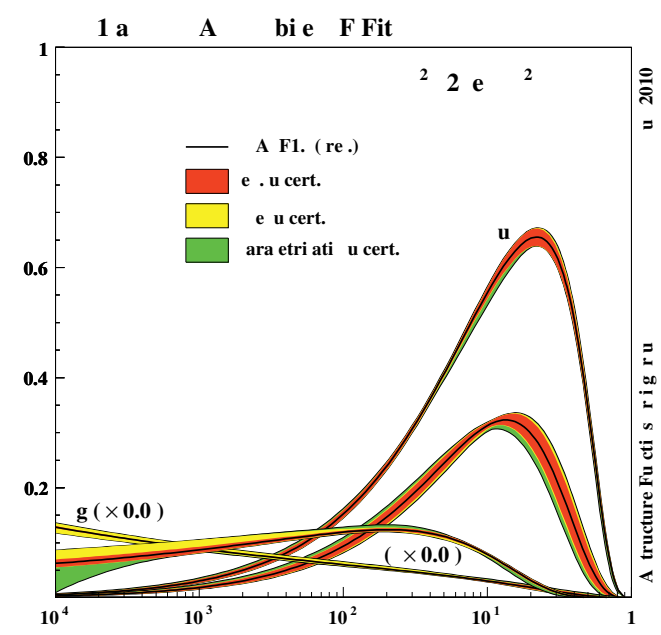

(b)

Figure 2. (a) The parton distribution functions from HERAPDF1.0, $x u_{v}, x d_{v}, x S=2 x(U+D), x g$, at $Q^{2}$ $=1.9 \mathrm{GeV}^{2}$. The gluon and sea distributions are scaled down by a factor 20. The experimental, model and parametrisation uncertainties are shown separately. (b) PDFs from HERAPDF1.5 (NLO) for the valence quarks, sea quarks and gluon at $Q^{2}=2 \mathrm{GeV}^{2}$ as a function of Bjorken- $x$. Uncertainties from experimental, model and parameterization sources are indicated.

Jet cross sections by ZEUS[6] in the photoproduction regime and by H1 in DIS[5] are shown in Figure 3(a) and 3(b) respectively. The ZEUS measurement takes into consideration different jet algorithms in an environment close to that of $p p$ collisions. H1's measurement corresponds to jet, dijet and trijet cross sections and uses an unfolding matrix which quantifies the statistical correlations between the measurement bins, thus providing an extra constraint for the PDF fits.

Charm measurements from H1 and ZEUS using different measurement techniques - D meson production, semi-leptonic decays and inclusive track measurements - are combined to give the $F_{2}^{c \bar{c}}$ structure function. This is used together with the combined inclusive data to extract the PDFs. The PDF fit was found to be clearly sensitive to the charm quark mass parameter $m_{c}^{\text {model }}$ when the charm data was included (Figure 4(b)) in contrast to the case when only inclusive data was used (Figure 4(a)) as seen by the $\chi^{2} /$ ndf of the PDF fit as a function of $m_{c}^{\text {model }}$. Different heavy flavour treatment schemes were found to have different optimum values of $m_{c}^{\text {model }}$ defined as that value giving the least $\chi^{2}$ from a scan of $m_{c}^{\text {model }}$, and are shown as the points in Figure 4(c). The predictions of $\mathrm{W}$ and $\mathrm{Z}$ cross sections at the LHC from the different heavy flavour treatments agreed considerably better at these optimized $m_{c}^{\text {model }}$ values, than at any arbitrarily chosen value, as shown for $\mathrm{Z}$ production in Figure 4(c). In this way the inclusion of the charm cross section data in the PDF fits helps to reduce the uncertainties on the $\mathrm{W}$ and $\mathrm{Z}$ cross sections. 


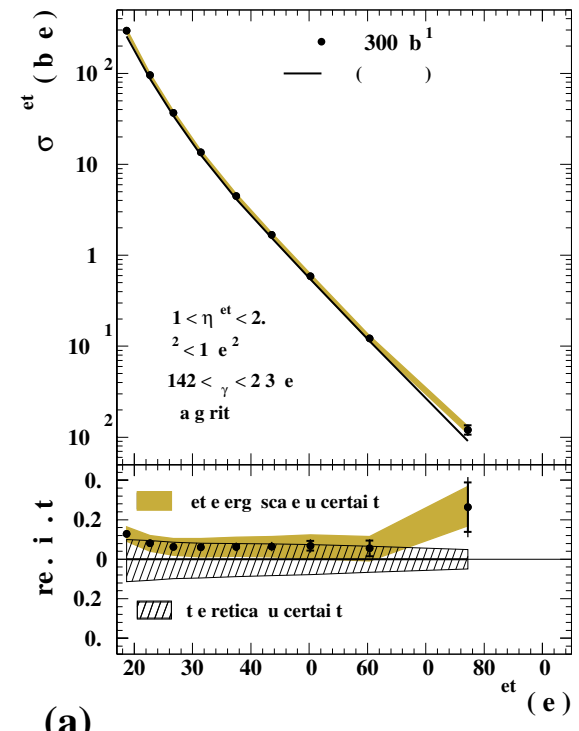

(a)

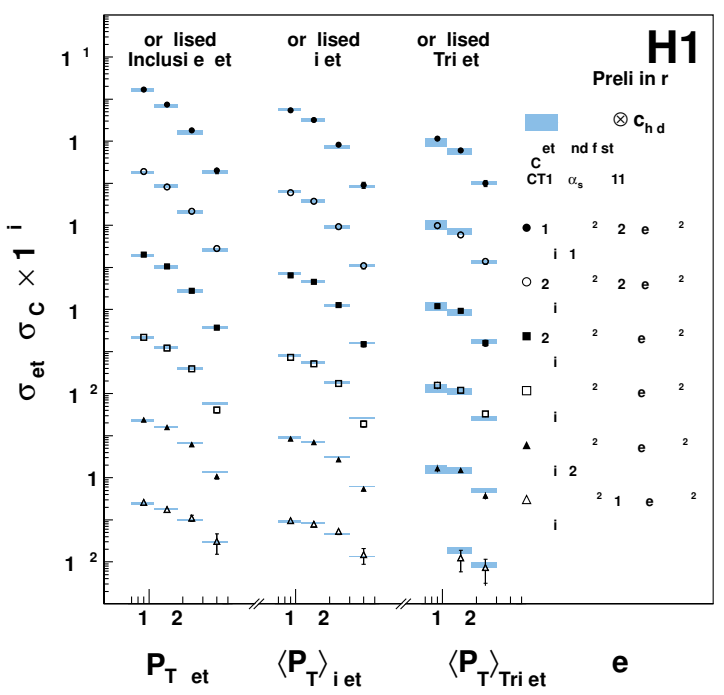

(b)

Figure 3. (a) ZEUS Jet cross section in photoproduction as a function of transverse jet energy. (b) H1 normalized jet, dijet and trijet cross sections in DIS as a function of transverse momentum for fixed ranges of $Q^{2}$.

\section{Conclusion}

Inclusive, jet and charm data have been measured at H1 and ZEUS. The inclusive and charm data have been combined and fitted into a parametrized proton to give precise PDFs. The final HERA PDFs are expected to highly impact our understanding of the proton and its associated $p p$ cross sectioins at the LHC. 


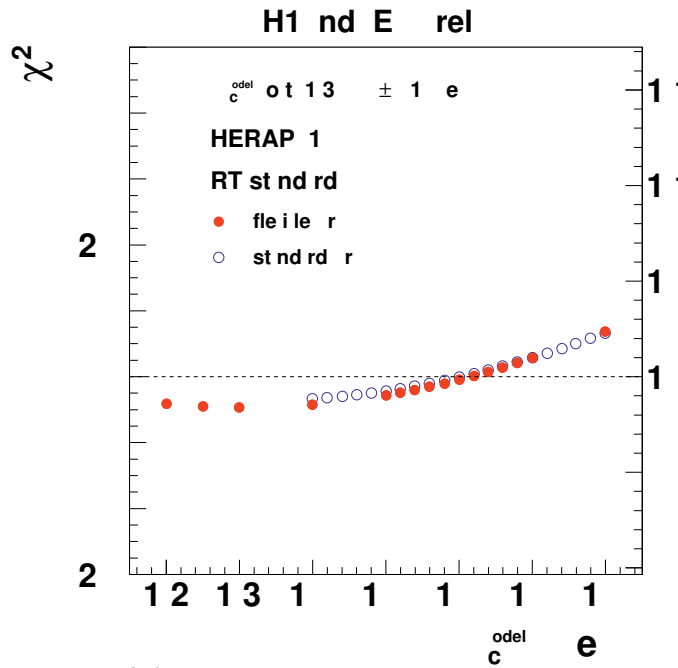

(a)

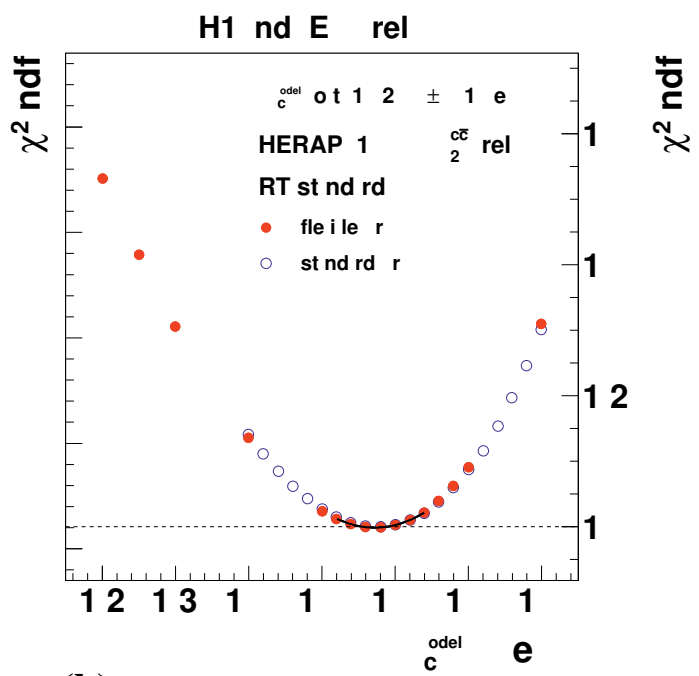

(b)

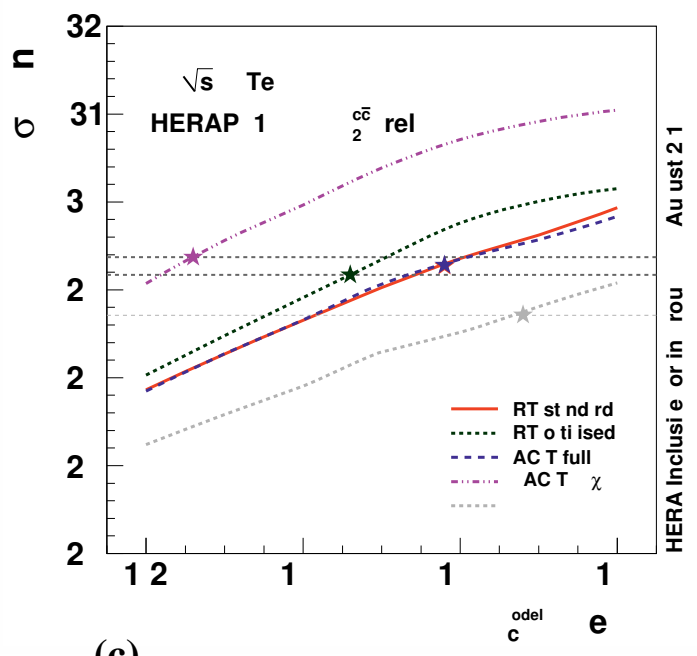

(c)

Figure 4. (a) $\chi^{2}$ of the HERA I data fit (HERAPDF1.0) in the standard RT scheme as a function of $m_{c}^{\text {model }}$. Open and closed symbols represent flexible and standard parametrisation repsectively. (b) $\chi^{2}$ of the HERA I $+F_{2}^{c \bar{c}}$ fit in the standard RT scheme as a function of $m_{c}^{\text {model }}$. Open and closed symbols represent flexible and standard parametrisation repsectively. (c) Total Z production cross section predictions at $\sqrt{s}=7 \mathrm{TeV}$ using PDFs derived from inclusive and charm data for different heavy flavour treatment schemes. The stars indicate the cross sections corresponding to the optimum value of the charm quark mass parameter $m_{c}^{\text {model }}$ for each treatment considered. 


\section{References}

[1] F. Aaron et al. [H1 Collaboration] (2009), [arXiv:0904.0929].

[2] F. D. Aaron et al. [H1 and ZEUS Collaboration], JHEP 1001, 109 (2010)

[3] H1 and ZEUS Collaborations, H1prelim-10-142, ZEUS-prel-10-018, 2010

[4] H1 and ZEUS Collaborations, H1prelim-11-042, ZEUS-prel-11-002, 2011

[5] H1 Collaboration, H1 prelim-12-031, 2012

[6] H. Abramowicz et al. [ZEUS Collaboration], Nucl. Phys. B 864, 1 (2012)

[7] H1 and ZEUS Collaborations, H1prelim-09-171, ZEUS-prel-09-015, 2009

[8] H1 and ZEUS Collaborations, H1prelim-10-143, ZEUS-prel-10-019, 2010

[9] Dokshitzer Y. L., Sov. Phys. JETP, 1977, 641-653

[10] Gribov V. N. and Lipatov L. N., Sov. J. Nucl. Phys., 1972, 438-450

[11] Gribov V. N. and Lipatov L. N., Sov. J. Nucl. Phys., 1972, 675-684

[12] Altarelli G. and Parisi G., Nucl. Phys., 1977, 298 